\title{
Atomic and Molecular Data and their Applications
}

\author{
Gordon W.F. Drake ${ }^{1, a}$, Jung-Sik Yoon ${ }^{2}$, Daiji Kato ${ }^{3}$, and Grzegorz Karwasz ${ }^{4}$ \\ ${ }^{1}$ Department of Physics, University of Windsor, Windsor, ON N9B 3P4, Canada \\ 2 Plasma Technology Research Center, National Fusion Research Institute, 37 Dongjangsan-ro, Gunsan, \\ Jeollabuk-do 54004, South Korea \\ 3 National Institute for Fusion Science, Oroshi-cho 322-6, Toki Gifu 509-5292, Japan \\ ${ }^{4}$ Institute of Physics, Nicolaus Copernicus University, ul. Grudziadzka 5/7, 87100 Torun, Poland
}

Received 8 January 2018

Published online 22 March 2018 - (c) EDP Sciences, Società Italiana di Fisica, Springer-Verlag 2018

\begin{abstract}
This topical issue on Atomic and molecular data and their applications was motivated by the 10th International Conference on Atomic and Molecular Data (ICAMDATA 2016), which was held from September 26 to 29, 2016 in Gunsan, Republic of Korea. The topics of this issue reflect those of the conference program. The scientific papers in the topical issue cover the fields of atomic and molecular structure, radiative transitions, scattering processes, data base development, and the applications of atomic and molecular data to plasma modeling.
\end{abstract}

\section{Introduction}

The Tenth International Conference on Atomic and Molecular Data (ICAMDATA 2016) was held in Gunsan, Republic of Korea from September 26 to 29, 2016. This was the latest in a continuing series of international conferences since 1997 that promotes the use of atomic and molecular (AM) data in various fields of science and technology. Most importantly, it provides a forum for interaction of AM data producers and users and for information exchange on AM data needs and availability. Its aim is to foster cross-disciplinary cooperation between the $\mathrm{AM}$ data producers and users, and to coordinate AM data activities and databases worldwide.

The field of atomic and molecular data covers a broad range of topics from the basic structure and spectroscopy of atoms, ions and molecules to the cross sections that control the rates of processes when these species collide and react in a hot gas or plasma, whether it be in a fusion reactor here on earth or in a remote astrophysical source. A detailed analysis of the light emitted by these sources contains a great wealth of information about the temperature, density and composition of the remote source. It is a knowledge of the microscopic processes taking place, such as the excitation, ionization of the atoms and molecules when the collide to form ions and free electrons, and their subsequent recombination and emission of light, that allows these connections and interpretations to be made through detailed computer models of the remote source.

\footnotetext{
${ }^{\star}$ Contribution to the Topical Issue "Atomic and Molecular Data and their Applications", edited by Gordon W.F. Drake, Jung-Sik Yoon, Daiji Kato, and Grzegorz Karwasz.

a e-mail: gdrake@uwindsor.ca
}

A second completely different area of application is not to hot gases, but to ultracold gases where that atoms and molecules can be brought nearly to rest in an atomic trap. Here, new quantum phenomena appear when the atomic species interact under highly controlled conditions, with potential applications to quantum computing.

The needs for atomic and molecular data are often met by direct laboratory measurements of the relevant transition frequencies, intensities and cross sections. However, such measurements are sometimes either not feasible or become very expensive. In that case, theoretical calculations from first principles, or extrapolations of known data, play an important role. Ideally, theory and experiment progress hand-in-hand, with each providing a check on the other. When the two agree, the process can be said to be well understood, and theory can be applied with confidence to other unknown cases. An estimation of the uncertainties in theoretical calculations has recently become an important topic, and a special panel discussion was devoted to that topic.

The three areas of focus for the conference therefore reflected these close connections between the needs for basic atomic and molecular data and their generation. The first area of focus was the experimental and theoretical atomic and molecular data generation. Topics included atomic and molecular structure and spectroscopy, electron collisions with atoms and molecules, heavy particle collisions, and particle-surface interactions. The last topic is especially important to an understanding of the interaction of a fusion plasma with the walls of its container.

The second area of focus was a survey of atomic and molecular data collections, assessment and dissemination. Since huge volumes of data are involved for the vast variety of processes that can take place, the organization and availability of the data become a key issue. Topics 
included data collection and selection, critical evaluation, and estimation of uncertainties; databases, data exchange and dissemination; and a survey of data center activities and standardization of data formats.

The third area of focus was the application and needs of atomic and molecular data to practical problems. Topics included astrophysics and atmospheric physics, magnetic and inertial fusion, laboratory and industrial plasmas, lighting science and technology, biomedicine and biophysics, combustion and environmental sciences and technology, surface physics, gaseous electronics, solid state optics, spectroscopy, and optoelectronics.

\section{Summary of contributions}

The papers published in this Topical Issue are representative of the papers presented and discussed at the conference. Beginning with basic atomic structure, the work of Ding et al. [1] on the energy levels and transition probabilities of the 54-times ionized tungsten ion $\mathrm{W}^{54+}$ shows that relativistic and quantum electrodynamic corrections make an important contribution in these heavy highly-ionized ions, and must be taken into account to get physically significant results. This result can generally be expected to be true for all heavy ions, and so comparisons with experiment are important. In this connection, Xu et al. [2] presented a new technique based on X-ray scattering at low momentum transfer to measure oscillator strengths and transition probabilities for valence electrons.

At the low-energy end of the spectrum, Zhang et al. [3] presented calculations of the long-range van der Waals interaction between lithium atoms and other noble gases, including spin-orbit effects for excited states of lithium. The results dominate the the behaviour of ultracold gases and Bose-Einstein condensates.

Two papers studied the effects of the plasma electric potential itself on atomic processes. In the simplest model problem, Hu et al. [4] calculated the effects of the plasma potential on the energy levels of highly charged hydrogenic systems. Laricchiuta et al. [5] considered the effects of plasma screening on resonant charge exchange in $\mathrm{H}+\mathrm{H}^{+}$ collisions. This provides a model of what to expect under extreme conditions of high density plasmas.

Of the 18 papers published as part of this Topical Issue, eight involve mainly theoretical basic collision processes that control the dynamical evolution of plasmas [6-13]. Some depend critically on detailed atomic structure calculations for inner shell excitations [6] followed by Auger processes to eject additional electrons (shake-off). This is the dominant mechanism for the creation of successively higher levels of ionization in a plasma. The inverse process of dielectronic recombination [7] is the dominant mechanism at low densities for ions and electrons to recombine in the absence of a third body. Also closely related are the calculations of resonantly enhanced excitation of copperlike gadolinium $\left(\mathrm{Gd}^{35+}\right)$ [8]. Spectral lines of gadolinium have been identified in a wide variety of astrophysical sources. The calculations of $\mathrm{Li}$ et al. [8] indicated that resonant enhancement can increase the cross sections by up to an order of magnitude.
Another process that depends critically on atomic structure is the electron impact ionization of argon atoms, particularly when the two outgoing electrons have equal energies. The calculations of Purohit and Singh [9] and $\mathrm{Hu}$ et al. [10] show that polarization of the residual $\mathrm{Ca}^{+}$ or $\mathrm{Ar}^{+}$ion by the electric field of the outgoing electrons critically affects the triply differential cross section. It is the TDCS that depends most critically on the details of the collision mechanism.

Three papers [11-13] tackled the much more difficult problem of electron scattering by molecules. The discussion of Fedus and Karwasz [11] pointed out the urgent need for improved calculations in view of the lack of agreement between theory and experiment, or theories with each other. As a benchmark for comparison at low and very low energies (down to meV) Kitajima et al. [12] have measured cross sections for electron scattering from $\mathrm{N}_{2}$. On the theoretical side, Gupta et al. [13] have obtained theoretical values for the ionization cross sections of the $\mathrm{C}_{2} \mathrm{~F}_{x}$ fluorocarbons, using the binary-encounter Bethe model. The results are especially important for the modelling of fluorocarbon plasmas.

The ultimate goal of this work is to apply the results to the modelling and diagnostics of plasmas. Three papers [14-16] reported progress in this area. Colonna et al. [14] presented a theoretical study of the response of the vibrational modes of $\mathrm{H}_{2}$ molecules to electrical pulses, including their coupling to the electronic and heavyparticle dynamics in a plasma. The aim is to probe the electron energy distribution in the plasma. Li et al. [15] studied the spectra produced by tantalum and hafnium in a laser-induced plasma, and identified the observed lines by comparison with theoretical calculations. Tantalum and hafnium are of particular interest as alternatives to tungsten in fusion devices. Murakami et al. [16] presented a detailed collisional-radiative model for $\mathrm{W}^{27+}$, including a comprehensive set of excitation and recombination processes for plasma diagnostics. Further to this work, Seon et al. [17] discussed progress on the design and development of an ITER diverter VUV spectrometer, particularly attuned to representative tungsten emission lines resulting from the tungsten diverter material. Its usefulness as a plasma diagnostic follows directly from the experimental and theoretical information on radiative transition probabilities.

Finally, Ryabchikova [18] discussed the accuracy required in atomic and molecular data for the determination of fundamental stellar parameters and chemical composition, galaxy chemical evolution, exoplanet formation, and reviewed the data bases available.

In addition to the regular scientific sessions, the conference included a panel discussion on standards and expectations for uncertainty estimates in theoretical calculations of atomic and molecular properties, to the extent that it is reasonably possible to do so. Since this has typically not been done in the past, there is a need for a paradigm shift in best practises for the field, now called uncertainty quantification (UQ) (for a recent discussion, see Ref. [19]). The participants were H.-K. Chung (IAEA), Gordon Drake (U. Windsor), Viatcheslav Kokoouline (U. Central Florida), Martin O'Mullane (Strathclyde), and 
Randall Smith (Harvard Smithsonian CFA). There was general agreement that there is an urgent need for better uncertainty quantification in order to make the results more useful for applications, and that this would elevate the importance of the field. Considerable progress has already been made in some areas such as atomic structure calculations, but uncertainties for scattering phenomena are in general much more difficult to handle. A definite policy to require uncertainty estimates in appropriate circumstances was recently announced by the journal Physical Review A [20].

\section{Epilogue}

The papers presented in this topical issue on atomic and molecular data and their applications bring together the experimentalists and theorists responsible for generating atomic and molecular data with the plasma modelers who make critical use of the data, and the people who build and maintain the extensive data bases to make the data available. The papers illustrate the key importance of uncertainty quantification for theoretical data in future developments of plasma modeling and applications. The papers reflect the broad international character of the field, and the opportunities provided for younger physicists and students to gain experience in this exciting area of research.

\section{References}

1. X. Ding, R. Sun, F. Koike, D. Kato, I. Murakami, H.A. Sakaue, C. Dong, Eur. Phys. J. D 71, 73 (2017)

2. L.-Q. Xu, Y.-W. Liu, X. Xu, D.-D. Ni, K. Yang, L.-F. Zhu, Eur. Phys. J. D 71, 183 (2017)
3. D.-H. Zhang, Y.-B. Xu, J. Jiang, L. Jiang, L.-Y. Xie, C.-Z. Dong, Eur. Phys. J. D 71, 175 (2017)

4. H.-W. Hu, Z.-B. Chen, W.-C. Chen, X.-B. Liu, N. Fu, K. Wang, Eur. Phys. J. D 71, 303 (2017)

5. A. Laricchiuta, G. Colonna, M. Capitelli, A. Sarim, B.M. Smirnov, Eur. Phys. J. D 71, 265 (2017)

6. R. Beerwerth, S. Fritzsche, Eur. Phys. J. D 71, 253 (2017)

7. L. Dou, L. Xie, D. Zhang, C. Dong, W. Wen, Z. Huang, X. Ma, Eur. Phys. J. D 71, 128 (2017)

8. S. Li, R. Si, C.-y. Zhang, X.L. Guo, Z.B. Chen, K. Wang, C.Y. Chen, J. Yan, Eur. Phys. J. D 71, 156 (2017)

9. G. Purohit, P. Singh, Eur. Phys. J. D 71, 143 (2017)

10. X.-Q. Hu, Z.-B. Chen, Y. Wang, K. Wang, Eur. Phys. J. D 71, $81(2017)$

11. K. Fedus, G.P. Karwasz, Eur. Phys. J. D 71, 138 (2017)

12. M. Kitajima, T. Kishino, T. Okumura, N. Kobayashi, A. Sayama, Y. Mori, K. Hosaka, T. Odagiri, M. Hoshino, H. Tanaka, Eur. Phys. J. D 71, 139 (2017)

13. D. Gupta, H. Choi, M.-Y. Song, G.P. Karwasz, J.-S. Yoon, Eur. Phys. J. D 71, 88 (2017)

14. G. Colonna, L.D. Pietanza, G.D. Ammando, R. Celiberto, M. Capitelli, A. Laricchiuta, Eur. Phys. J. D 71, 279 (2017)

15. B. Li, T. Otsuka, E. Sokell, P. Dunne, G.O. Sullivan, H. Hara, G. Arai, T. Tamura, Y. Ono, T.-H. Dinh, T. Higashiguchi, Eur. Phys. J. D 71, 278 (2017)

16. I. Murakami, A. Sasaki, D. Kato, F. Koike, Eur. Phys. J. D 71, 246 (2017)

17. C. Seon, J. Hong, I. Song, J. Jang, H. Lee, Y. An, B. Kim, T. Jeon, J. Park, W. Choe, H. Lee, S. Pak, M. Cheon, J. Choi, H. Kim, W. Biel, P. Bernascolle, R. Barnsley, M.O. Mullane, Eur. Phys. J. D 71, 313 (2017)

18. T. Ryabchikova, Eur. Phys. J. D 71, 169 (2017)

19. H.-K. Chung, B.J. Braams, K. Bartschat, A.G. Csaszar, G.W.F. Drake, T. Kirchner, V. Kokoouline, J. Tennyson, J. Phys. D: Appl. Phys. 36, 363002 (2016)

20. The Editors, Phys. Rev. A 83, 040001 (2011) 